\title{
Deformation and Stress of a Composite-Metal Assembly
}

\author{
Chensong Dong* \\ Department of Mechanical Engineering, Curtin University, GPO Box U1987, Perth, WA 6845, \\ Australia \\ Lu Kang \\ RCR Tomlinson Ltd., 21 Wilson Road, Bunbury WA 6230, Australia
}

\begin{abstract}
Compliant structures e.g. automobile body panel and airplane wing box are widely used. A compliant structure consists of one or more flexible parts, and these parts share the mating features among them. Because of process-induced deformation and part-to-part variations, external forces are applied during the assembly process and the parts are deformed. As a result, the final assembly is pre-stressed and its geometrical shape may deviate from the designed shape. Therefore, the assembly variation and residual stress need to be analysed in order to evaluate the structure performance.

In this study, a new approach based on Response Surface Methodology (RSM) is developed. A number of organised virtual experiments are conducted with the aid of Finite Element Analysis (FEA) and regression models are fitted to the resulting data. These regression models relate part variations to assembly variation and residual stress. Monte Carlo simulation can be conveniently done using these simple regression models. The effectiveness of this method was illustrated using a composite-metal assembly. It is shown that the method presented in this paper provides a practical and reliable solution to the analysis of compliant structures.
\end{abstract}

Keywords: Compliant; assembly; deformation; tolerance; stress

\section{Introduction}

Compliant structures e.g. automobile body panel and airplane wing box are widely used. A compliant structure consists of one or more flexible parts, and these parts are sharing the mating features among them. Because of process-induced deformation and part-to-part variations, external forces are applied during the assembly process and the parts are deformed. As a result, the final assembly is pre-stressed and its geometrical shape may deviate from the designed shape. Therefore, the assembly variation and residual stress need to be analysed in order to evaluate the structure performance.

Compliant mechanisms are widely used for micro-motion [1]. For example, a compliant mechanical amplifier was design by Ouyang et al [2]. Most studies on compliant structures, however, are focused on sheet metal assembly process. Liu and $\mathrm{Hu}[3][3]$ developed a compliant assembly model to analyse the effect of deformation and spring-back on assembly deviation by applying liner mechanics and statistics. Liu et al. [4][4] performed a variation analysis for assemblies in parallel and in series, respectively. It is indicated that for a simple compliant

\footnotetext{
* Corresponding author. Tel: +61 (8) 92669204; fax: +61 (8) 92662681; email: c.dong@curtin.edu.au.
} 
assembly which consists of two pieces of sheet metal, the spring-back force is equal to the sum of clamping forces in magnitude but opposite in direction.

Camelio et al. [5][5] extended this model to multi-station systems using state space representation by incorporating sources of variation from parts, tooling and their interactions. It is suggested that if two or more spring-back forces are induced on an assembly, the sum of spring-back forces is identical to the sum of revered clamping forces and corresponding reaction forces. By using this relationship, the assembly process and-can be simulated with the aid of upfront Finite Element Analysis (FEA). External clamping forces can be applied on the nominal part/assembly model with the directions reversed, and virtual variations can be created.

Based on general assembly process, the overall variation in an assembly can be studied by certain variation analysis methods, e.g. Worst Case (WC) method, Root Sum Squares (RSS) method and Monte Carlo simulation.

When the WC method is used, the assembly variation is found by adding the maximum/minimum variations of the parts $[6,7][6,7]$. A more realistic variation analysis is known as the RSS method, which is based on Uncertainty Propagation Law. When using RSS, the assembly variation is assumed to be normally distributed. When the major concern is finding the statistical distribution of assembly variation, Monte Carlo simulation can be employed.

For compliant assemblies, direct Monte Carlo simulation is infeasible because it requires FEA for each simulation and is too time-consuming. Method of Influence Coefficients is often applied. The key to this method is to conduct mechanistic simulation for each part and assembly with the nominal geometric shape. Based on the constant linear force-displacement relationship obtained, the stiffness matrices of the parts and assembly can be derived. By Inversing the stiffness matrix of the assembly, and multiplying the sum of the stiffness matrices of the parts, the system sensitivity matrix can be obtained. The part and assembly variations are linked by this sensitivity matrix, and the mechanistic variation model is then established. By using Monte Carlo simulation, the distribution of the assembly variation can be generated. Method of Influence Coefficients is an efficient analytical method for enclosed complex sheet metal assembly with multiple parts. Lee et al. [8][8] applied Method of Influence Coefficients on the study of the wing-box assembly of a commercial airplane. The study also indicated that Method of Influence Coefficients should be used with care only when the assembly variation is much smaller than the source variations because the stiffness matrix of the assembly actually varies with the geometrical shape. If the assembly has very large deformation compared to the source variations, the change in the cross section of the assembly will be significant. In such case, the stiffness matrices obtained from the constant linear relationship will be no longer valid. Another limitation of Method of Influence Coefficients is that for a compliant assembly consisting of orthotropic materials such as composites, the linear relationship in is difficult to find. In such case, Method of Influence Coefficients is difficult to apply. A recent study by Tonks et al. [9][9] showed that covariant statistical model can be applied to the compliant structure analysis without deriving the mechanistic variation model.

It is seen from the literature that for compliant structures, part variations have significant impact on the assembly variation, and it is important to establish their relationship. Traditional variation analysis methods such as the WC and RSS are not applicable for compliant assemblies since they are based on rigid body assumption-. Method of Influence Coefficients is suitable for enclosed sheet metal structure but it is not easy to use in an orthotropic material made assembly. The literature survey shows few studies have been carried out for the assembly of composite parts. In this study, a new approach based on Response Surface Methodology (RSM) is developed. A number of organised virtual experiments are conducted with the aid of FEA and regression models are fitted to the resulting data. These regression models relate part variations to assembly variation 
and residual stress. Monte Carlo simulation can be conveniently done using these simple regression models.

The method presented in this study is of general usefulness because it provides an efficient and organised way to tolerance analysis of assemblies, especially when the materials are complex, e.g. composites, and linear relationships are difficult to find.

\section{Methodology}

For a compliant structure, when there is no process-induced deformation, all components have perfect geometric shapes as designed, and they will fit perfectly in the assembly. However, in practice, process-induced deformation and part-to-part variations are unavoidable. Thus, external forces are needed to bring the deformed parts back to their nominal shapes in the assembly process. After external forces are removed, deformation and residual stresses are induced.

As shown in Figure 1, the assembly process of compliant structures consists of four steps. In step 1, the enclosed angles decrease due to process-induced deformation, which can be characterised by the angular deflections $\theta_{1}$ and $\theta_{2}$, respectively. During assembly, part 1 is mounted on to the rigid jig and part 2 is placed on its top. The jig is considered much stiffer than the parts so that the deformation of the jig can be neglected. The jig is designed to have the nominal shape, so that clamping forces are needed to fit part 1 to the jig. In step 2, clamping forces $F_{1}$ and $F_{2}$ which are normal to the inclined surfaces are applied. When clamping forces are increased, the gaps between part 1 and the jib decreases until the gaps become zero. In step 3, the flat plate (part 2) is placed on the top of the part 1. These two components are bonded together with glue or mechanical fasteners. It is assumed that no stress is induced by glue or mechanical fasteners. In step 4, after part 1 and part 2 are completely assembled, the clamping forces (including the reaction forces exerted on part 1) are removed and the assembly is then removed from the jig. Spring-back and residual stresses are induced.

Because the assembly deformation is complex, closed-form solutions for assembly deformation and residual stresses are difficult to find. Therefore, upfront FEA is needed. In this study, first individual parts in an assembly are considered. The force or pressure that causes process-induced deformation is derived. The force or pressure for each individual part is then applied to the nominal assembly model, and the assembly deformation and residual stresses can be derived.

In order to obtain statistical distribution of assembly deformation, direct Monte Carlo simulation with the aid of FEA is time-consuming and infeasible. In this study, an alternative approach based on RSM [10][10] is presented. The common application for response surface methodology is optimizing the design parameter to maximize the system output result through serials of designed experiments. The purpose of using response surface methodology in this work is using a sequence of proper designed experiments to establish the relationship between the part deformation and assembly deformation without calculating sensitivity matrices. First, the process-induced deformation of each part is treated as input factors. Several levels are chosen for each input factor and the factor combinations are chosen in a certain way. For each factor combination, upfront FEA is conducted and the assembly deformation is found. In addition, the maximum residual stress is also obtained. Using the assembly deformation and maximum residual stress are as the responses to the input, a second order polynomial model is developed to reflect the relationship between the input factors and responses.

With the aid of the developed regression models, the distributions of assembly deformation and residual stresses can be obtained by Monte Carlo simulation. Random part deformation is generated and input into the regression models, and the corresponding assembly deformation and 
maximum residual stress are calculated. It should be noted that the regression models from RSM are only an approximating model and do not physically reflect the true functional relationship for an actual assembly. However, they are useful to predict the response when the mechanism (how the part deformations affect assembly) is unknown.

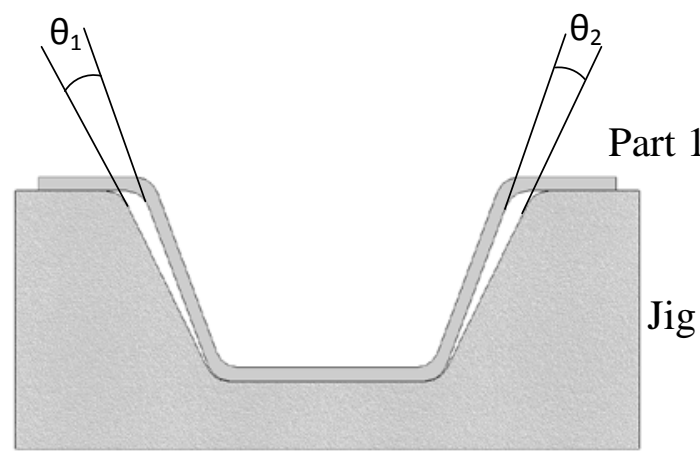

(a)

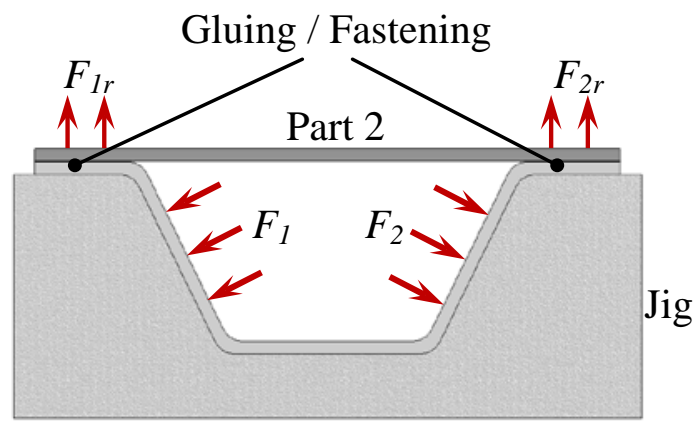

(c)

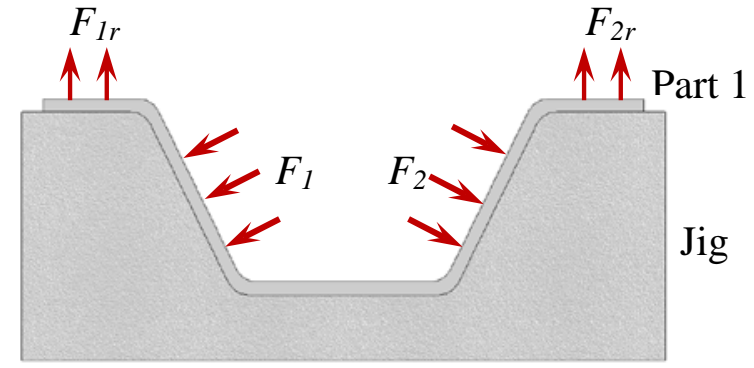

(b)

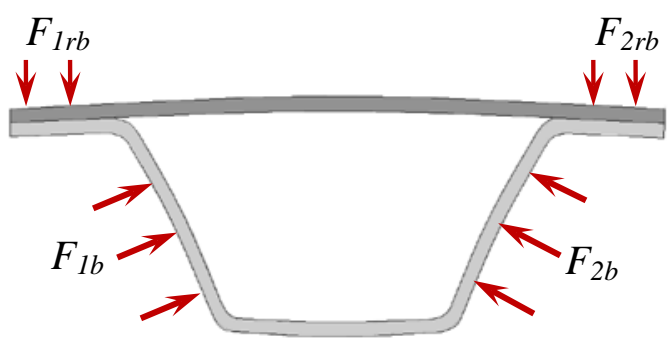

(d)

Figure 1:Assembly process of a compliant structure: (a) Mount the deformed part onto the jig; (b) Apply clamping forces to fit the part to the jig; (c) Mount the top plate on part 1; (d) Remove the assembly from the jig

\section{Case Study}

A compliant assembly consisting of a composite reinforcing rib being glued to an aluminium panel, as shown in Figure 2, is used to illustrate the methodology developed in this study. The depth and thickness of the assembly are $300 \mathrm{~mm}$ and $3 \mathrm{~mm}$, respectively. The composite reinforcing rib is made of AS4 graphite fibre and epoxy resin, and its sectional dimensions are shown in Figure 3. Because of heating and chemical reactions, spring-in, i.e. the reduction in an enclosed angle is induced. The process-induced spring-in affects the structure assembly. If a rigid jig is assumed, after the composite reinforcing rib is mounted onto the jig, clamping forces are needed to achieve the desired assembly. After the clamping forces are released, residual stresses and assembly spring back deformation are induced.

When the fibre volume fraction $50 \%$ and the stacking sequence $[0 / 90]_{\mathrm{s}}$ were assumed, and the processing and room temperature were $150^{\circ} \mathrm{C}$ and $20^{\circ} \mathrm{C}$, the spring-in was calculated using the effective CTE (coefficients of thermal expansion) by a simple mathematical formula. 


$$
\Delta \phi=\frac{\left(\alpha_{T}-\alpha_{I}\right)\left(T_{0}-T_{c}\right)}{1+\alpha_{T}\left(T_{0}-T_{c}\right)}(180-\phi)
$$

where $\alpha_{\mathrm{I}}$ and $\alpha_{T}$ are the in-plane and through-thickness effective CTE, respectively, and $\phi$ is the enclosed angle. The CTE were assumed to be independent of temperature.

The nominal spring-in from the calculation is $\Delta \phi_{1}=\Delta \phi_{2}=-0.67^{\circ}$ and $\Delta \phi_{3}=\Delta \phi_{4}=-0.40^{\circ}$.

In order to understand the influence of part variations, sequence of experiments was conducted with the aid of centre composite design (CCD). The spring-in $\Delta \phi$ at each corner radius was regarded as an independent process factor. For each process factor, two coded levels -1 and +1 were chosen, and then the coded centre level 0 and two additional levels $\pm \alpha$ were added. In this study, for simplicity, the nominal values were used as the centre levels. There is no blocking that splits the experiments since FEA simulation is conducted instead of doing actual experiments. Thus, all the design points at the same distance from the centre point should have the same prediction standard error. Therefore, rotatable CCD was selected. The value of $\alpha$ for rotatable CCD is calculated to be $2[10][10]$.

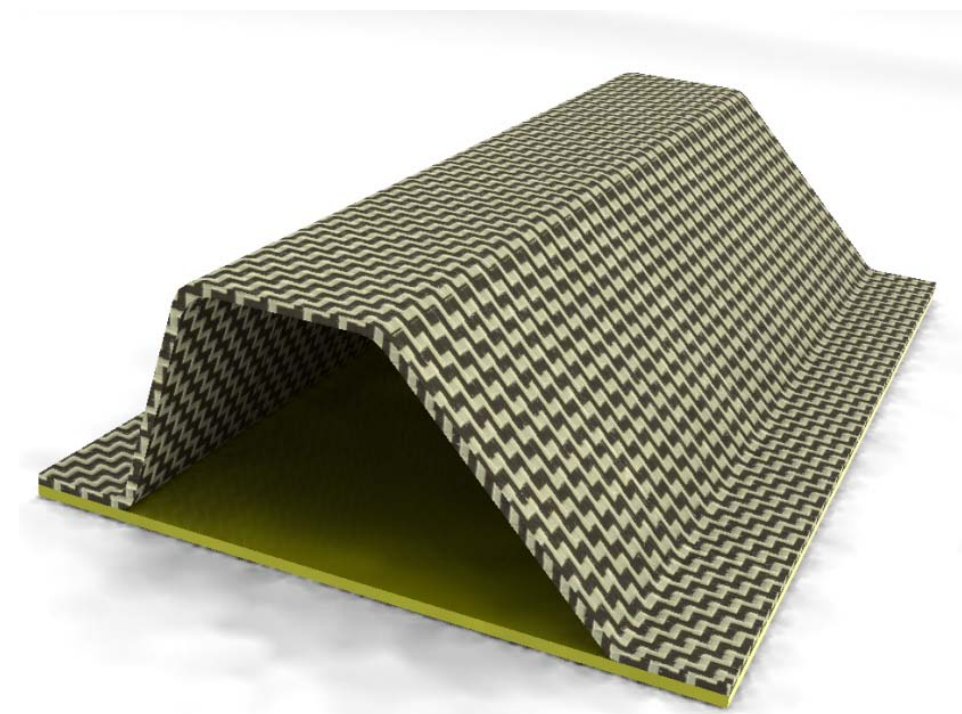

Figure 2: Composite reinforcing rib-aluminium panel assembly

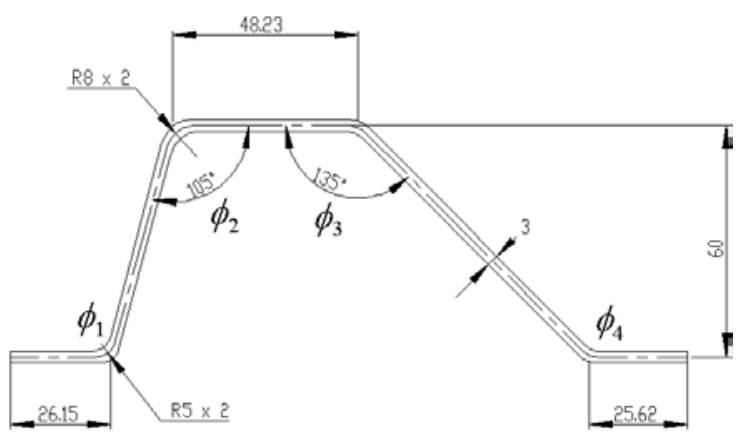

Figure 3: Dimensions of composite reinforcing rib (mm)

The finite element model for the composite reinforcing rib in the nominal dimensions was first built in ANSYS, as shown in Figure 4. SOLSH190 element was used. Pressure was applied on the four flat areas to cause required spring-in, as shown in Figure 5. The pressure values were found using the pressure-displacement relationships. 
Table 1: Actual and code levels of process factors

\begin{tabular}{|c|c|c|c|c|c|}
\hline \multirow{2}{*}{ Process factor } & \multicolumn{5}{|c|}{ Coded level } \\
\hline & -1 & 0 & +1 & $-\alpha$ & $+\alpha$ \\
\hline$\Delta \phi_{1}\left(^{\circ}\right)$ & -0.33 & -0.67 & -1.00 & 0 & -1.34 \\
\hline$\Delta \phi_{2}\left(^{\circ}\right)$ & -0.33 & -0.67 & -1.00 & 0 & -1.34 \\
\hline$\Delta \phi_{3}\left(^{\circ}\right)$ & -0.20 & -0.40 & -0.60 & 0 & -0.80 \\
\hline$\Delta \phi_{4}\left(^{\circ}\right)$ & -0.20 & -0.40 & -0.60 & 0 & -0.80 \\
\hline
\end{tabular}

Figure 4: Finite element model for composite reinforcing rib

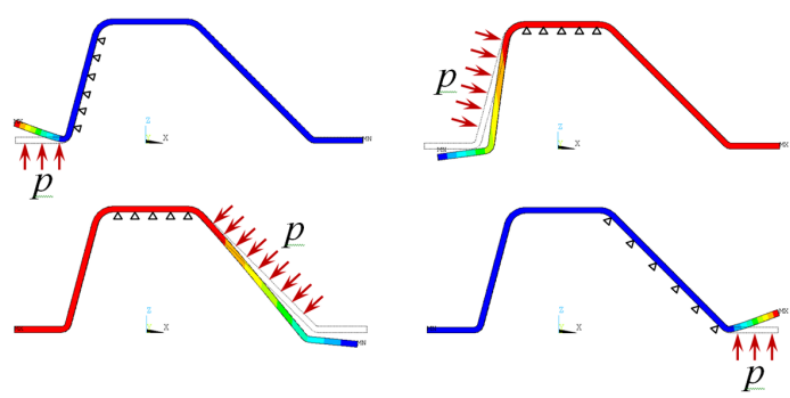

Figure 5: Pressure applied causing spring-in

For each combination of factors, the corresponding pressure found previously was applied onto the assembly model, as shown in Figure 6, and the assembly deformation and residual stress were computed with the aid of FEA. In this study, the residual stress due to assembly is represented by von Mises stress.

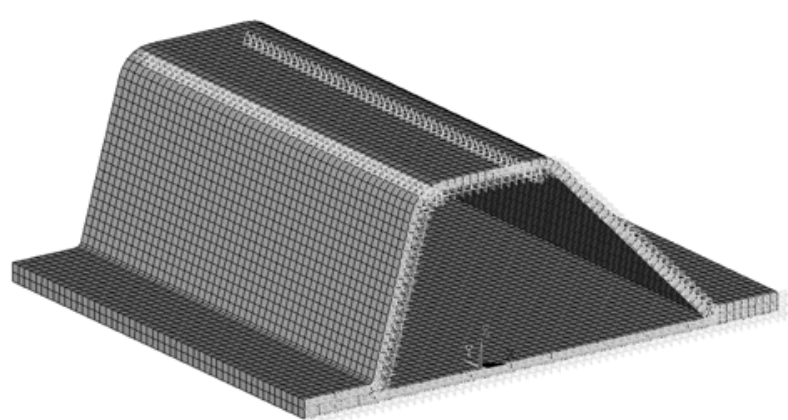

Figure 6: Finite element model for assembly 
When $\Delta \phi_{1}=1.00^{\circ}, \Delta \phi_{2}=0.33^{\circ}, \Delta \phi_{3}=0.20^{\circ}$ and $\Delta \phi_{4}=0.20^{\circ}$, the assembly deformation and residual stress are shown in Figure 7. It is seen high stresses are induced at the corner radii.

Two critical characteristics were used to describe the deformed shape. As shown in Figure 7, the deformation of the aluminium panel is complex. The surface geometric change was represented by geometric tolerance flatness. On the top surface of the composite reinforcing rib, the centre line was chosen as the reference axis, and position tolerance parallelism was chosen to evaluate the position of the aluminium panel with regard to the reference axis. Figure 8 illustrates the flatness and parallelism.

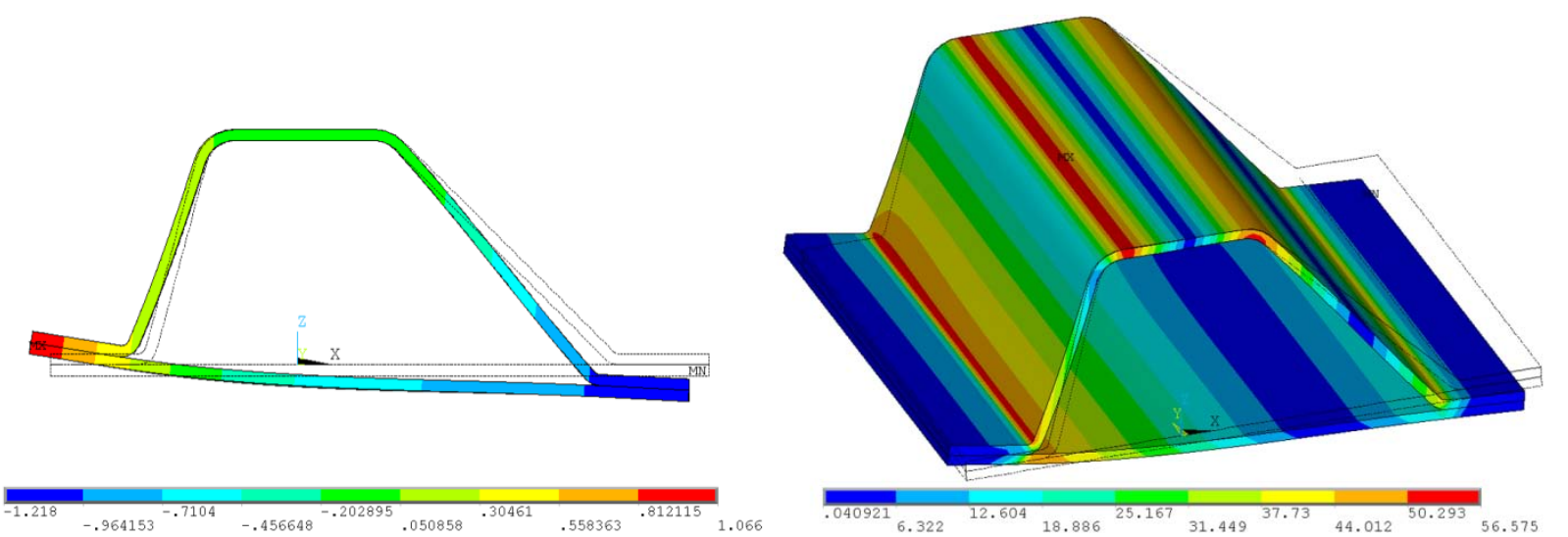

Figure 7: Assembly deformation (left) and residual stress (right) when $\Delta \phi_{1}=1.00^{\circ}, \Delta \phi_{2}=0.33^{\circ}, \Delta \phi_{3}=0.20^{\circ}$ and $\Delta \phi_{4}=0.20^{\circ}$

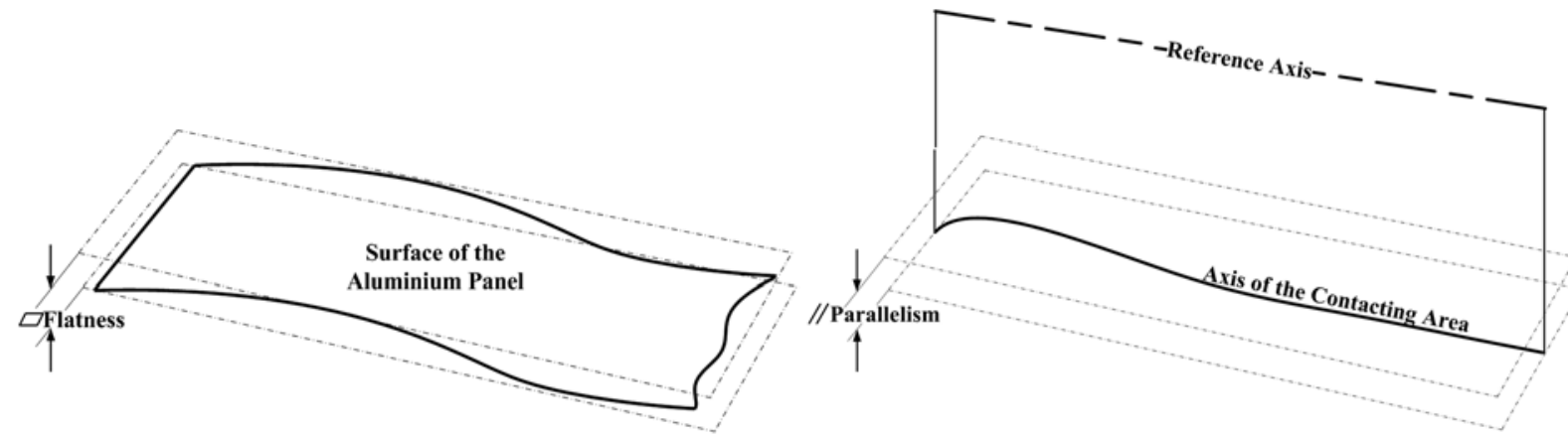

Figure 8: Flatness (left) and parallelism (right)

As shown in Figure 9, two parallel lines are constructed to enclose the profile, and the distance between these two straight lines is the flatness. The parallelism is simply the difference between the maximum and minimum $z$-displacements.

The complete result for all the 25 combinations is shown in Table 2. Regression models were fitted to the data as shown in Table 2 by using the stepwise regression technique. Box-Cox transformation was used to stabilize variance and make the data more normal distribution-like. The final regression models are:

$$
\begin{aligned}
\delta_{F}^{2.5}= & 0.03488-0.2195 \Delta \phi_{1}-0.02025 \Delta \phi_{2}-0.01808 \Delta \phi_{3}-0.03434 \Delta \phi_{4}+0.6034 \Delta \phi_{1}^{2}+1.9686 \Delta \phi_{4}^{2} \\
& +0.1434 \Delta \phi_{1} \Delta \phi_{3}-1.1589 \Delta \phi_{1} \Delta \phi_{4}+0.2542 \Delta \phi_{2} \Delta \phi_{4}-0.1070 \Delta \phi_{3} \Delta \phi_{4}
\end{aligned}
$$




$$
\begin{aligned}
\delta_{P}^{2}= & -2.018 \times 10^{-3}+6.394 \times 10^{-3} \Delta \phi_{1}+1.067 \times 10^{-4} \Delta \phi_{2}+1.126 \times 10^{-3} \Delta \phi_{3}-3.785 \times 10^{-3} \Delta \phi_{4} \\
& +26.054 \Delta \phi_{1}^{2}+0.9198 \Delta \phi_{2}^{2}+0.6091 \Delta \phi_{3}^{2}+178.563 \Delta \phi_{4}^{2}-9.794 \Delta \phi_{1} \Delta \phi_{2}+7.993 \Delta \phi_{1} \Delta \phi_{3} \\
& -136.423 \Delta \phi_{1} \Delta \phi_{4}-1.501 \Delta \phi_{2} \Delta \phi_{3}+25.637 \Delta \phi_{2} \Delta \phi_{4}-20.920 \Delta \phi_{3} \Delta \phi_{4}
\end{aligned}
$$

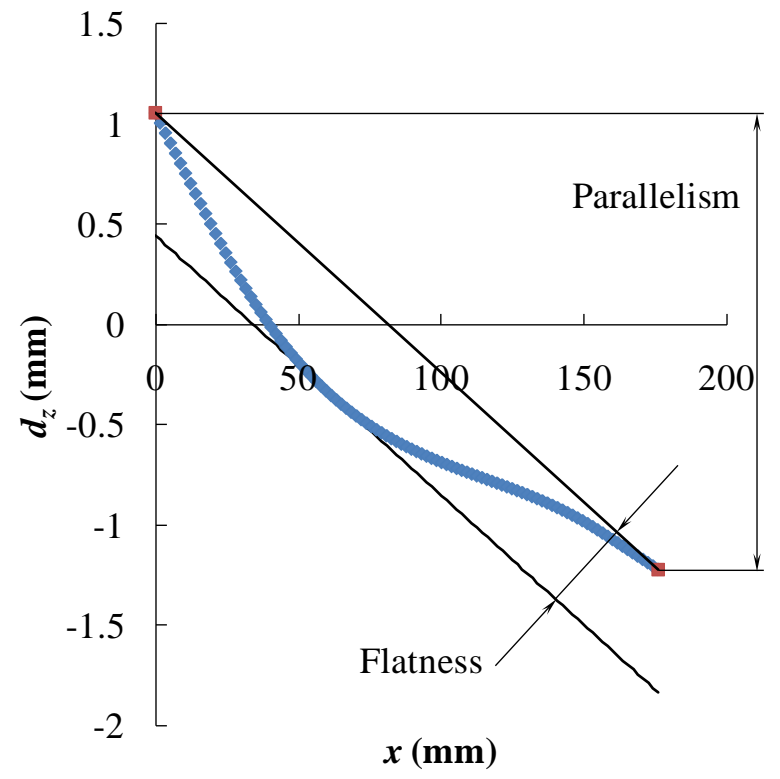

Figure 9: Calculation of flatness and parallelism

Table 2: Complete result for all the 25 combinations

\begin{tabular}{cccccccc}
\hline $\begin{array}{c}\text { Standard } \\
\text { order }\end{array}$ & $\Delta \phi_{1}\left(^{\circ}\right)$ & $\Delta \phi_{2}\left(^{\circ}\right)$ & $\Delta \phi_{3}\left(^{\circ}\right)$ & $\Delta \phi_{4}\left({ }^{\circ}\right)$ & $\begin{array}{c}\text { Flatness } \\
(\mathrm{mm})\end{array}$ & $\begin{array}{c}\text { Parallelism } \\
(\mathrm{mm})\end{array}$ & $\begin{array}{c}\text { Maximum residual } \\
\text { stress (MPa })\end{array}$ \\
\hline 1 & 0.33 & 0.33 & 0.20 & 0.20 & 0.27 & 1.14 & 39.60 \\
2 & 1.00 & 0.33 & 0.20 & 0.20 & 0.61 & 2.28 & 56.58 \\
3 & 0.33 & 1.00 & 0.20 & 0.20 & 0.32 & 1.78 & 59.46 \\
4 & 1.00 & 1.00 & 0.20 & 0.20 & 0.63 & 1.63 & 60.45 \\
5 & 0.33 & 0.33 & 0.60 & 0.20 & 0.29 & 0.82 & 34.18 \\
6 & 1.00 & 0.33 & 0.60 & 0.20 & 0.64 & 2.59 & 66.73 \\
7 & 0.33 & 1.00 & 0.60 & 0.20 & 0.34 & 1.46 & 51.18 \\
8 & 1.00 & 1.00 & 0.60 & 0.20 & 0.66 & 1.95 & 58.34 \\
9 & 0.33 & 0.33 & 0.20 & 0.60 & 0.77 & 6.50 & 168.03 \\
10 & 1.00 & 0.33 & 0.20 & 0.60 & 0.74 & 3.08 & 108.08 \\
11 & 0.33 & 1.00 & 0.20 & 0.60 & 0.83 & 7.14 & 190.80 \\
12 & 1.00 & 1.00 & 0.20 & 0.60 & 0.79 & 3.73 & 126.74 \\
13 & 0.33 & 0.33 & 0.60 & 0.60 & 0.76 & 6.18 & 159.48 \\
14 & 1.00 & 0.33 & 0.60 & 0.60 & 0.76 & 2.77 & 105.36 \\
15 & 0.33 & 1.00 & 0.60 & 0.60 & 0.82 & 6.83 & 182.25 \\
16 & 1.00 & 1.00 & 0.60 & 0.60 & 0.81 & 3.41 & 118.79 \\
17 & 0.00 & 0.67 & 0.40 & 0.40 & 0.66 & 5.69 & 147.16 \\
18 & 1.34 & 0.67 & 0.40 & 0.40 & 0.82 & 1.14 & 90.14 \\
19 & 0.67 & 0.00 & 0.40 & 0.40 & 0.49 & 1.63 & 68.46 \\
20 & 0.67 & 1.34 & 0.40 & 0.40 & 0.59 & 2.92 & 97.99 \\
21 & 0.67 & 0.67 & 0.00 & 0.40 & 0.52 & 2.59 & 87.15 \\
22 & 0.67 & 0.67 & 0.80 & 0.40 & 0.56 & 1.96 & 71.24 \\
\hline
\end{tabular}




\begin{tabular}{lllllllc}
\hline 23 & 0.67 & 0.67 & 0.40 & 0.00 & 0.49 & 3.09 & 72.01 \\
24 & 0.67 & 0.67 & 0.40 & 0.80 & 0.95 & 7.64 & 204.62 \\
25 & 0.67 & 0.67 & 0.40 & 0.40 & 0.54 & 2.27 & 79.19 \\
\hline
\end{tabular}

$$
\begin{aligned}
\sigma_{\max }^{2}= & 6.8875 \times 10^{2}-4.8660 \times 10^{3} \Delta \phi_{1}+1.5172 \times 10^{3} \Delta \phi_{2}-1.3640 \times 10^{3} \Delta \phi_{3}+2.8776 \times 10^{3} \Delta \phi_{4} \\
& +1.8301 \times 10^{4} \Delta \phi_{1}^{2}+1.0452 \times 10^{5} \Delta \phi_{4}^{2}-7.0361 \times 10^{3} \Delta \phi_{1} \Delta \phi_{2}+5.3821 \times 10^{3} \Delta \phi_{1} \Delta \phi_{3} \\
& -7.0979 \times 10^{4} \Delta \phi_{1} \Delta \phi_{4}+1.9129 \times 10^{4} \Delta \phi_{2} \Delta \phi_{3}-1.2756 \times 10^{4} \Delta \phi_{3} \Delta \phi_{4}
\end{aligned}
$$

Monte Carlo simulation was conducted using the developed regression models. It is assumed that $\Delta \phi_{1}$ and $\Delta \phi_{2}$ follow $N(0.665,0.112)$ and $\Delta \phi_{3}$ and $\Delta \phi_{4}$ follow $N(0.40,0.0667)$. The normal distributions were chosen based on what have been found in experiments. A pseudorandom number generator in MATLAB2009 was employed to generate these spring-in angles, and the corresponding flatness, parallelism and maximum residual stress were calculated. The resulting distributions are shown in Figure 10.
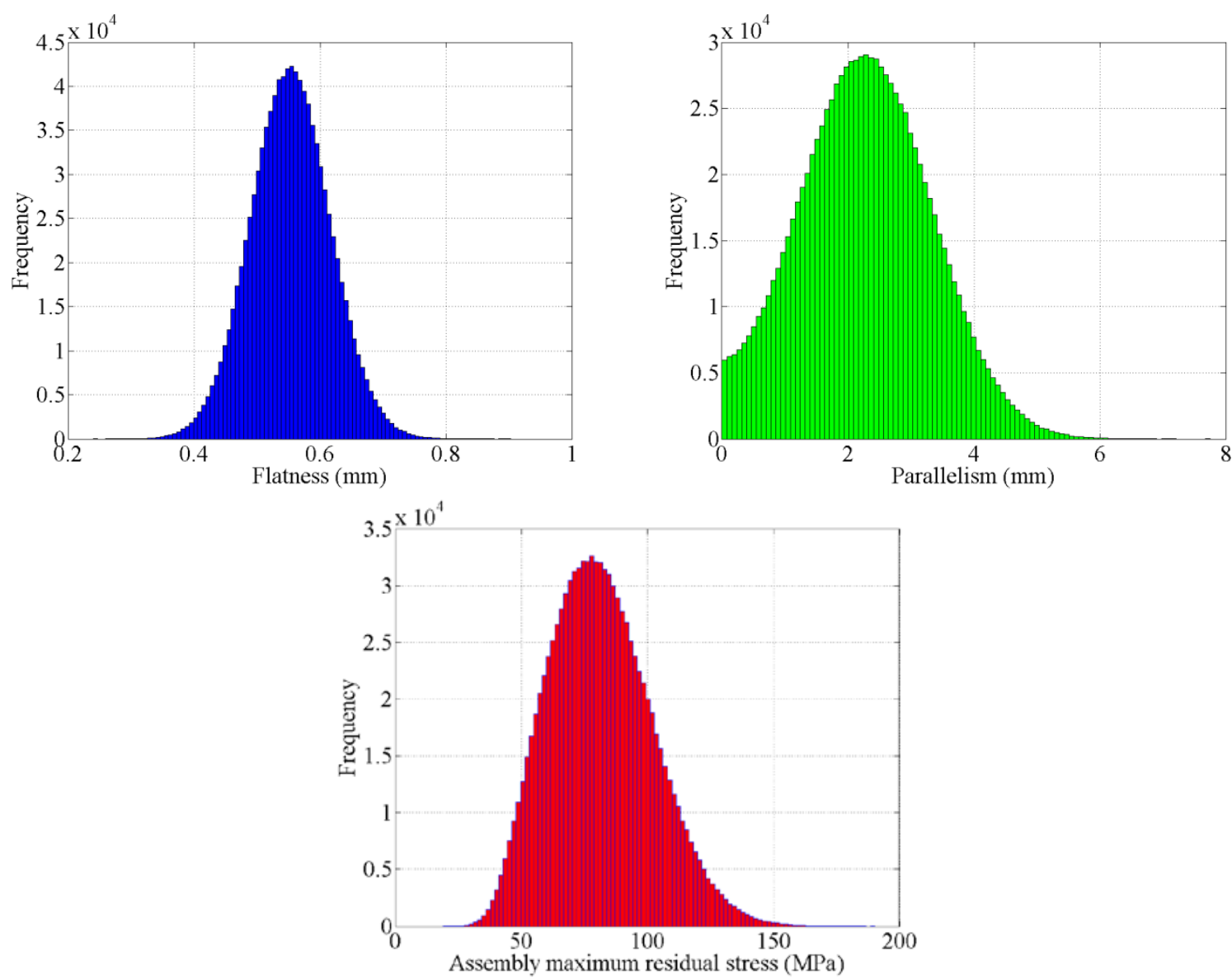

Figure 10: Statistical distributions from Monte Carlo simulation. Clockwise from top left: flatness; parallelism; assembly maximum residual stress

It is seenseems that flatness is normally distributed but parallelism and stress areis not. The distribution of maximum residual stress is slightly skewed but can be regarded as normal. For the purpose of comparison, the uncertainties of the assembly deformation and stress were also evaluated using the RSS. A comparison of the RSS and Mote Carlo simulation is given in Table 3. It is seen that these two methods give very similar standard deviations but very different mean 
values. The main reason is the RSS assumes the system output being normally distributed but this is not always true. Therefore, Monte Carlo simulation gives more reliable results.

Table 3: Comparison of RSS and Mote Carlo simulation

\begin{tabular}{lcccc}
\hline & \multicolumn{2}{c}{ Mean } & \multicolumn{2}{c}{ Standard deviation } \\
\cline { 2 - 5 } & RSS & $\begin{array}{c}\text { Monte Carlo } \\
\text { simulation }\end{array}$ & RSS & $\begin{array}{c}\text { Monte Carlo } \\
\text { simulation }\end{array}$ \\
\hline Flatness (mm) & 0.18 & 0.55 & 0.06 & 0.06 \\
Parallelism (mm) & 3.18 & 2.28 & 1.06 & 1.04 \\
Maximum residual stresses (MPa) & 64.23 & 81.40 & 21.41 & 20.48 \\
\hline
\end{tabular}

\section{Conclusions}

In this study, a new approach based on RSM is developed. A number of organised virtual experiments are conducted with the aid of FEA and regression models are fitted to the resulting data. These regression models relate part variations to assembly variation and residual stress. Monte Carlo simulation can be conveniently done using these simple regression models.

A composite-metal assembly was used to illustrate the effectiveness of this method. The assembly deformation was characterised by flatness and parallelism. It is seen that the statistical distributions of assembly deformation and residual stress can be conveniently obtained by using the RSM based regression models. For the parallelism and assembly residual stress, significant non-normal distributions are found. Therefore, the method presented in this paper provides a practical and reliable solution to the analysis of compliant structures.

In the practical applications, the distributions of component variations are first determined, and then their levels can be properly chosen. The FEA can be done and regression models can be developed. With these regression models the distributions of assembly deformation and residual stresses can be obtained using Monte Carlo simulation.

\section{Acknowledgment}

Chensong Dong thanks the support from the Curtin Research Fellowship.

\section{References}

1. Ouyang, P.R., et al., Micro-motion devices technology: the state of arts review. Journal of Advanced Manufacturing Technology, 2008. 38(5-6): p. 463-478.

2. $\quad$ Ouyang, P.R., W.J. Zhang, and M.M. Gupta, Design of a new compliant mechanical amplifier, in 2005 ASME Design Engineering Technical Conference. 2005: Long Beach, CA, USA.

3. Liu, S.C. and S.J. Hu, Variation simulation for deformable sheet metal assemblies using finite element methods. ASME Journal of Manufacturing Science and Engineering, 1997. 119(3): p. 368-374.

4. Liu, S.C., S.J. Hu, and T.C. Woo, Tolerance analysis for sheet metal assemblies. ASME Journal of Mechanical Design, 1996. 118(1): p. 62-67. 
5. Camelio, J., S.J. Hu, and D. Ceglarek, Modeling variation propagation of muti-station assembly system with compliant parts. ASME Journal of Mechanical Design, 2003. 125(4): p. 673-681.

6. Chase, K.W. and W.H. Greenwood, Design issues in mechanical tolerance analysis. Manufacturing Review, ASME, 1988. 1(1): p. 50-59.

7. Dong, Z., W. Hu, and D. Xue, New production cost-tolerance models for tolerance synthesis. ASME Journal of Engineering for Industry, 1994. 116: p. 119-206.

8. Lee, B., et al., Variation analysis of three dimensional non-rigid assemblies, in Proceedings of the 2007 IEEE International Symposium on Assembly and Manufacturing. 2007, IEEE: Ann Arbor, Michigan, USA. p. 13-18.

9. Tonks, M.R., K.W. Chase, and C. Smith, Predicting deformation of compliant assemblies using covariant statistical tolerance analysis, in Models for Computer Aided Tolerancing in Design and Manufacturing, J.K. Davidson, Editor. 2007, Springer Netherlands. p. 321-330.

10. Montgomery, D.C., Design and analysis of experiments, 7th edition. 2009: John Wiley \& Sons, Inc. 